\title{
Modeling the Dynamics of the Cardiovascular-respiratory System (CVRS) in Humans, a Survey
}

\author{
F. Kappel ${ }^{1,2,3 *}$ \\ ${ }^{1}$ Center for Quantitative Sciences in Biomedicine, North Carolina State University \\ Raleigh, NC 27695-8213 \\ ${ }^{2}$ Center for Research in Scientific Computation, North Carolina State University \\ Raleigh, NC 27695-8212 \\ ${ }^{3}$ Institute for Mathematics and Scientific Computation, University of Graz \\ A 8010 Graz, Austria
}

\begin{abstract}
In this paper we give a survey on modeling efforts concerning the CVRS. The material we discuss is organized in accordance with modeling goals and stresses control and transport issues. We also address basic modeling approaches and discuss some of the challenges for mathematical modeling methodologies in the context of parameter estimation and model validation.
\end{abstract}

Keywords and phrases: cardiovascular-respiratory system, blood flow, parameter estimation

Mathematics Subject Classification: 76Z05, 92C35, 93A30

\section{Introduction}

In the following we give a survey on modeling issues concerning different aspects of the CVRS. We start in Section 2 with a short description of the tasks which have to be accomplished by this system and continue in Section 3 with a discussion of the primary goals pursued in modeling processes in the context of the CVRS. In particular we will discuss goals concerning control processes. In Section 4 we present some basic modeling principles used for the CVRS, stressing modeling by lumped parameter models. Finally, in Section 5 we emphasize some of the challenges for mathematical methodologies caused by the fact that on the one hand models of increasing complexity have to be developed in order to meet the requirements posed by clinical applications and on the other hand we are confronted with a shortage of data for model validation. An additional factor is the necessity to develop patient specific models for clinical applications. We want to point out that also this survey is a modeling process. Consequently, whatever is said in the following is a more or less simplified version of the real situation. For the basics in physiology of the cardiovascular and respiratory systems we refer to $[34,39,49,52,54,86,99]$.

${ }^{*}$ Corresponding author. E-mail: franz.kappel@uni-graz.at 


\section{Tasks of the CVRS}

The cardiovascular system (CVS) is the central transport system in the human body responsible, in cooperation with the respiratory system (RS), for supplying tissues and organs with $\mathrm{O}_{2}$ and substrates required for metabolism and, at the same time, removing $\mathrm{CO}_{2}$ and other end-products of metabolism. In addition, the CVS is responsible for the distribution of hormones and other substances in the body. As examples we mention the distribution of insulin which is secreted by the $\beta$-cells in the Langerhans-islets of the pancreas and is distributed to the cells in the liver, muscle, and fat tissue to take up glucose from the blood and storing it as glycogen. In case of need the stored sugar is used as an energy source through glycogenolysis, which breaks down the stored glycogen into glucose ([40, pp. $961-978])$. In order to accomplish these tasks the CVS needs to be connected with the outside world. This is provided by a number of other systems and organs, as for instance the digestive systems, the kidneys and the RS which governs the exchange of the blood gases $\mathrm{O}_{2}$ and $\mathrm{CO}_{2}$, i.e., $\mathrm{O}_{2}$ is entering into blood for distribution by the CVS and $\mathrm{CO}_{2}$ collected by the CVS is eliminated from the body. Since a proper functioning of the exchange of the blood gases needs a tight coordination between blood flow and ventilation in the lungs, where this exchange takes place, the RS and the CVS are intimately connected also concerning the control actions. Therefore, frequently it is necessary to consider the combined CVRS as one system [15] (compare also Subsection 3.1).

\subsection{The CVS}

The transport of substances which have to be distributed to different parts of the body is accomplished by blood flow through the vessels of the two circuits of the CVS, the systemic and the pulmonary circuit (compare [11,12]). Blood is the transport medium for the CVS where the substances which have to be transported are dissolved in plasma as for instance a major part of $\mathrm{CO}_{2}$ or bound to carrier molecules as for instance $\mathrm{O}_{2}$ which is bound to the hemoglobin of the red blood cells [48, Chapter 16]. The necessary blood flow is generated by the heart which can be considered as a serial arrangement of two pulsatile pumps. The systemic circuit is that part of the CVS which actually distributes the substances in the body, whereas the pulmonary circuit together with the lungs primarily is responsible for the exchange of the blood gases $\mathrm{O}_{2}$ and $\mathrm{CO}_{2}$. The first vessel of the systemic circuit is the aorta which receives blood from the left ventricle. Then the blood stream is distributed by the systemic arteries branching from larger to smaller arteries till the arterioles, the smallest arteries before the capillaries. Leaving the capillary region blood is collected by the venules and reaches through progressively larger veins the right heart. Microcirculation refers to blood flow in the micro-vessels, the arterioles, the capillaries and the venules [90]. An important function of the arterioles is to adjust blood flow to tissues and organs according to metabolic needs. This is accomplished by dilation respectively constriction of these vessels which very efficiently changes flow resistance in these vessels and consequently leads to a redistribution of blood flow. The venous part of the systemic circuit because of the high compliance of its vessels is also a reservoir for blood which can be mobilized when needed. The capillaries play a key role for the exchange between blood and interstitium. In the pulmonary circuit the right heart is pumping blood into the pulmonary arteries which branch repeatedly till the capillaries reach the alveoli in the lungs where the exchange of blood gases between blood and inhaled air takes place $\left(\mathrm{CO}_{2}\right.$ leaves and $\mathrm{O}_{2}$ enters blood). Then blood is collected by the pulmonary veins and enters the left heart.

\subsection{The RS}

The RS provides the link of the CVS with the surrounding air. It consists of the air passages (nose, mouth, pharynx, larynx) and the broncheal trees through which inhaled air finally reaches the alveoli, which are terminal sacs surrounded by capillaries. The airflow is either into the alveoli (inhaling) or out of the alveoli (exhaling) and is referred to as ventilation. The walls of the alveoli is made up of the respiratory membrane which is made up of a dense network of capillaries. This provides a large area of contact between air and blood which enables the exchange of the blood gases by diffusion (see [48, 
Chapter 17], [13], [43, Chapter 6]). The ventilation is governed by the respiratory muscles. As already mentioned at the end of the introduction to Section 2 the ventilation and the blood flow have to be adjusted precisely in order to guarantee an optimal gas exchange.

\section{Modeling goals}

As in any modeling process, also when modeling in the context of the CVRS it is absolutely necessary to have a clear picture of the goals to be achieved eventually. This in general is a non-trivial requirement which needs intensive consideration by the team involved in the modeling process and is in particular important, because the type of the mathematical model to be developed depends to a large degree on the modeling goals. One could term this the principle of priority of the practical problem. Of course, most of the goals listed in the following have also implications for model based diagnostic procedures. In this context we want to draw attention to [85], [55].

\subsection{Control issues}

Because of the multitude of tasks the CVRS has to accomplish and the fact that, despite frequently occurring perturbations, a number of variables has to be kept within narrow limits, it is clear that a large number of hierarchically organized and intertwined control loops is necessary for the proper working of this system (see for instance [44], [43, Chapter 5]).

The following, certainly incomplete list demonstrates the wide range of control issues considered in the literature and gives an idea how complex regulation of the CVRS is:

- Reaction of the CVS/CVRS to an ergometric workload (for a comprehensive discussion see [83]). In this case the main aspect is to satisfy the metabolic needs for delivering a workload imposed on the system. In $[46,47],[15$, Section 1.4] the fact that in case of a constant modest workload the CVS reaches a new equilibrium situation (which can be obtained from measurements) was used to formulate an optimal control problem which provides the stabilizing feedback control. Instead of the linear-quadratic regulator problem considered in [47] also nonlinear feedback laws can be obtained (see [15, Appendix B]). A promising approach is to use receding horizon controls which may be less sensitive to perturbations and non-modeled dynamics [57]. In case of a non-constant ergometric workload these approaches are not applicable. The question arises if there are quantities associated with the CVS which are controlled to nominal values in wide range of different situations. The state variables used in models for the CVS are certainly not candidates for such a quantity. However, in the CVRS the partial pressure $P_{a, \mathrm{CO}_{2}}$ of $\mathrm{CO}_{2}$ and the $\mathrm{pH}$-value in blood are such candidates, the nominal values being $\sim 40 \mathrm{mmHg}$ for $P_{a, \mathrm{CO}_{2}}$ and $\sim 7.35-7.45$ for the $\mathrm{pH}$ value. Using a combined model for the CVRS (as presented in [15, Chapter 3]) an optimal control was obtained for nonconstant ergometric workloads in [41] by using the Euler-Lagrange formulation of the optimal control problem. In addition, the metabolic need for $\mathrm{O}_{2}$ and the metabolic production of $\mathrm{CO}_{2}$ had to be matched to some extend with $\mathrm{O}_{2}$ - and $\mathrm{CO}_{2}$-transport by blood. This supports the approach by G. Fink (see [32]) that the metabolic demands of organs and tissues control the circulation and blood pressure, for instance, is an emergent quantity determined by the goal to satisfy the metabolic demands.

- Reaction of the CVS/CVRS to orthostatic stress. This refers to the impact of gravity or other forces leading to a redistribution of blood volume in the different parts of the CVS. For instance, a postural change from supine to standing upright causes a volume shift of about $0.5-0.7$ liters from the central part of the body to the lower part. As a consequence, filling of the ventricles and consequently cardiac output as well as blood flow to the brain are reduced, which may lead to syncope. Counter measures of the CVRS aim at an increase of cardiac output (increased heart frequency, increased contractility and mobilization of unstressed volume in order to increase the filling pressure for the ventricles). Standard tests to assess the reaction of the CVS/CVRS to orthostatic stress are the sit-to-stand test, the headup-tilt test (HUT) and the lower-body-negative-pressure test (LBNP) (see [14,42, 45, 64, 65]). A very interesting mechanism concerns mental activity and its influence on the reaction of the CVS-controls 
to orthostatic stress [37]. For instance, the "mental activity" considered in [37] was to conduct simple arithmetic calculations during the orthostatic stress situation. Intimately connected with the problem of orthostatic stress are the strong autoregulatory mechanisms of cerebral blood flow which have been investigated in $[60,61,63]$, for instance.

- Reaction of the CVS/CVRS to a loss of blood volume in the CVS. This control issue is related to the previous one. The difference is that also the loss of blood volume has to be compensated. We mention two special cases:

- Hemorrhage. The consequences of a loss of blood depend crucially on the volume of blood which has been lost. Small amounts can be compensated by capillary refill. If the volume of blood lost is not too large then a restitution of volume by saline solution or the like can be sufficient. However, if too much blood was lost then the oxygen carrying capacity of blood has to be restored in order to avoid irreparable damage of organs (compare [10,59]). A modeling goal could be to provide help to assess the status of a patient who has suffered hemorrhage of unknown degree.

- Volume changes during dialysis. For patients with end stage renal disease undergoing hemodialysis treatment at each dialysis session excess fluid has to be removed. This is done by choosing a proper ultrafiltration rate in the dialyzer. This involves assessment of the fluid status of the patient during the session and control of blood volume in order to avoid hypovolemia, but also to achieve a proper fluid status at the end of the session (see [15], [11, Section 4.6] and [19-23,95,96]).

- Model based defibrillation. In this case the models are usually 3D-models of cardiac tissue involving the so-called bidomain equation describing the electrical properties of heart tissue. The problem is to interfere with an electric countershock with sufficient intensity at the right phase (compare $[17,58,89])$.

\subsection{Transport issues}

The central function of the CVS for distribution of all kinds of substances requires an understanding of the relevant aspects of blood flow in the system of vessels of the CVS. Besides the geometric complexity of cardiovascular system we have to deal with blood which is not a Newtonian fluid but a suspension of blood cells (erythrocytes, leukocytes, platelets) in plasma (see [15, Subsection 4.5.7]). There is a large number of efforts to model blood flow in large parts of the CVS as well as for specific details. Blood flow in the arterial tree is considered in [62], for instance. In order to deal with flow problems in vessels with moving boundaries compliance of vessels which result in moving boundaries C. S. Peskin developed the so-called immersed boundary method in [73]. Moving boundaries are a consequence of compliant vessel walls or flexible respectively moving structures embedded in the blood flow (heart valve leaflets or artificial heart valves) (see also [74-78] and [56]). Interaction of viscous fluid flow and elastic vessel walls was considered in [24]. Patient specific modeling in this context in particular means that one also has to include the individual geometry of vessels and other components into the model. This involves mesh generation on the basis of information obtained from MRI (see for instance [87], [97]). Considerable efforts have been invested into blood flow problems in coronary arteries in order to provide assistance for bypass surgery (compare $[1,71]$ ).

Dependent on the modeling goals one may have to model the component of the CVS which is of central interest (for instance the left ventricle) in its full 3-D geometry and must also include a model for the blood flow in adjacent components (for instance in the aorta), because of important flow interactions in the two components. However, it may be sufficient to consider a 1-D model for these adjacent components. This raises the problem of dealing with geometric multi-scale models (see for instance $[25,35,69]$ ).

A complex problem which involves both, transport issues but also control issues is drug delivery in the human body. It is clear that in this context transport phenomena are very important. In particular the microcirculation (see Subsection 2.1) plays an important role, because in case of a subcutaneous administration the drug enters the systems via microcirculation and in the majority of cases it is also delivered via microcirculation to the organ or tissue which is targeted. The problem is that blood flow in the larger vessels is rather different from that in the microcirculation, the reason being that in larger vessels blood can be considered as viscous Newtonian fluid. However, this is definitely not possible in 
very small vessels, because blood is a suspension of blood cells in plasma and consequently a two-phase flow develops in small vessels where the cells move faster along the center line of the vessel than plasma skimming along the vessel walls [15, Subsection 4.5.7]. This results in a much lower apparent viscosity of blood in the small capillaries compared to that in larger vessels. This the Fahraeus-Lindquist effect [30]. For a geometric multi-scale approach coupling microcirculation with flow in larger arteries see [51]. For coupling a 1-D model of arteries with a 0-D model for the microcirculation see [2]. Control issues become very important in connection with closed loop drug delivery systems (intravenous infusion of vasodilators or anesthetics, for instance, see [88]).

The pulsatile nature of blood flow which is particularly pronounced in the arterial parts of the systemic and the pulmonary circuit is frequently approximated by a non-pulsatile flow in global compartment models for the CVS (see [5] for a comparison of pulsatile and non-pulsatile flow). Likewise the ventilation in compartment models for the RS and the CVRS is frequently replaced by a continuous unidirectional flow of air. Of course, there is a number of phenomena where these simplifications in the models are not permitted. Compare for instance [92-94] where the connection between pulsatility of blood flow and baroreceptor action is considered.

Another class of modeling problems is related to plaque development at vessel walls. High-density and low-density lipoproteins (HDL and LDL) in blood transport cholesterol to locations where it is needed. LDL can move through vessel walls leaving the cholesterol they carry there. It is clear that besides this mechanism also the local blood flow situation plays an important role for plaque development (compare $[80,82]$ and $[72])$. A somewhat similar situation concerns the deposition of platelets and fibrin at damaged vessel walls, for instance near stents (see $[3,53]$ ).

\section{Modeling of the CVRS}

In this section we want to discuss some of the basic approaches towards modeling components of the CVRS respectively the whole system. In doing this we concentrate on lumped parameter models or compartment models which are well represented in the literature (see, for instance, $[15,18,67,68,98]$ ).

The CVRS is a 3-D system of high complexity. However, 3-D models are appropriate only if the phenomena which are the modeling goals can only be characterized in a 3-D setting as, for instance, flow problems associated with certain components of the CVRS (compare the problems discussed in Subsection 3.2). Control issues as presented in Subsection 3.1 are usually investigated using compartment models where the functionally different parts of the system are lumped to a compartment. In doing this the basic assumption is that the parameters associated with the components which are included in the compartment can be replaced by parameters for the compartment so that the dynamics of the compartment in the model is representing the overall dynamics of the components which are merged into the compartment with sufficient accuracy.

A global compartment model for the CVS would distinguish the compartments according to Figure 1. The arterial systemic compartment, for instance, lumps together all the arteries of the systemic circuit. Since the vessels of the CVS have elastic walls the arterial systemic, the venous systemic and the two pulmonary compartments are so-called compliant vessels which are characterized by the volume $V$ and the (transmural) pressure $P$ in the vessel which are related by

$$
V=f(P) .
$$

The function $f$ is the capacitance of the vessel. The volume $V_{u}=f(0)$ which is the volume in the vessel corresponding to transmural pressure 0 is called the unstressed volume of the vessel. Mobilization of unstressed volume is an important component in the reaction of the CVS to orthostatic stress (compare [45]). In many models the pressure-volume relation is simplified to $V=c P+V_{u}$ respectively to $V=c P$, where the constant $c$ is called the compliance of the vessel. Referring to the introduction of this section we remark that, for instance, the compliance $c_{\mathrm{as}}$ of the arterial systemic compartment is a replacement of the compliances for the arteries included in this compartment which are very different. Therefore the parameter $c_{\text {as }}$ cannot be measured directly but has to be identified on the basis of available data. 


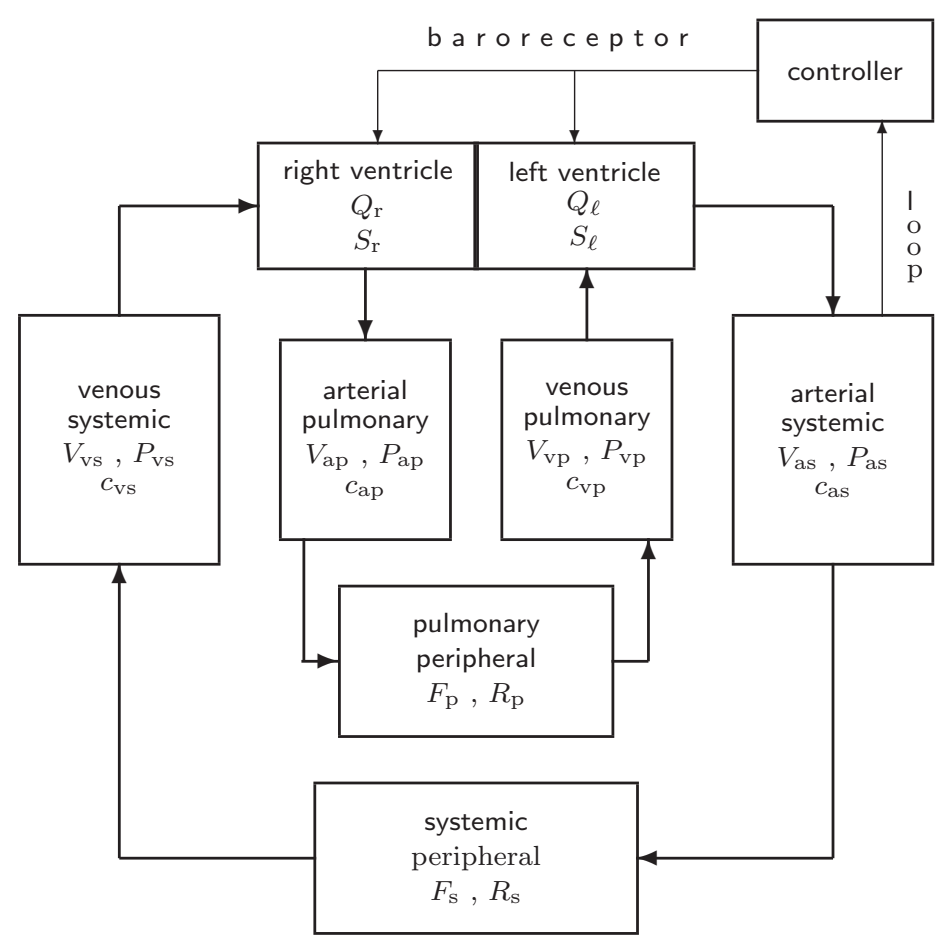

FiguRE 1. Block-diagram of a compartment model for the CVS.

In Figure 1 we see also the systemic and pulmonary peripheral regions which are characterized by the resistance $R$ against blood flow across the region. The systemic peripheral region comprises the arterioles connecting it with the systemic arteries, the capillary regions embedded in the organs and tissues and the venules connecting with the veins of the venous systemic compartment. The pulmonary peripheral region is built essentially by the capillaries surrounding the alveoli of the lungs. These two compartments are examples of a resistance vessel where the blood flow $F$ across the region is usually expressed by Ohm's law,

$$
F=\frac{1}{R}\left(P_{1}-P_{2}\right)
$$

where $P_{1}$ and $P_{2}$ are the pressures in the adjacent compartments. In case of the systemic peripheral region the resistance $R_{\mathrm{s}}$ is controlled by local autoregulatory mechanisms in order to adjust the blood flow to the metabolic needs of organs and tissues. In such a case $R_{\mathrm{s}}$ is not a parameter of the system but a state variable.

In order to get a dynamic model for the global CVS one has to describe the change of volume respectively of pressure in the compartments of the system. For the compliance vessels it is clear that the total change of volume is the difference between inflow and outflow which for the arterial systemic compartment gives

$$
\frac{d}{d t} V_{\mathrm{as}}=Q_{\ell}-F_{\mathrm{s}}, \quad F_{\mathrm{s}}=\frac{1}{R_{\mathrm{s}}}\left(P_{\mathrm{as}}-P_{\mathrm{vs}}\right) .
$$

Using the simplified pressure volume relation we obtain

$$
c_{\mathrm{as}} \frac{d}{d t} P_{\mathrm{as}}=Q_{\ell}-F_{\mathrm{s}} .
$$


In a analogous fashion we get differential equation for the other three compliance vessels:

$$
\begin{aligned}
c_{\mathrm{as}} \frac{d}{d t} P_{\mathrm{as}} & =Q_{\ell}-F_{\mathrm{s}}, \\
c_{\mathrm{vs}} \frac{d}{d t} P_{\mathrm{vs}} & =F_{\mathrm{s}}-Q_{\mathrm{r}}, \\
c_{\mathrm{ap}} \frac{d}{d t} P_{\mathrm{ap}} & =Q_{\mathrm{r}}-F_{\mathrm{p}}, \\
c_{\mathrm{vp}} \frac{d}{d t} P_{\mathrm{vp}} & =F_{\mathrm{p}}-Q_{\ell} .
\end{aligned}
$$

It remains to derive expressions for the cardiac outputs $Q_{\ell}$ and $Q_{\mathrm{r}}$. This was done by considering the ventricles as compliance vessel for the filling process and using Frank-Starling's law of the heart for the ejection process. For this we refer to [15, Chapter 1], [46,47] where the reaction of the CVS to a constant ergometric workload was considered. In this context the important control loop is the baroreceptor loop. In [47] this was modeled as

$$
\dot{H}=u(t),
$$

where $u(t)$ was determined as a feedback control using the linear-quadratic regulator problem.

The model (4.1) is usually called Grodin's model of the mechanical part of the CVS. Adding the equations (4.1) we get

$$
\frac{d}{d t}\left(c_{\mathrm{as}} P_{\mathrm{as}}+c_{\mathrm{vs}} P_{\mathrm{vs}}+c_{\mathrm{ap}} P_{\mathrm{ap}}+c_{\mathrm{vp}} P_{\mathrm{vp}}\right) \equiv 0,
$$

i.e., the total blood volume $V_{0}=c_{\mathrm{as}} P_{\mathrm{as}}+c_{\mathrm{vs}} P_{\mathrm{vs}}+c_{\mathrm{ap}} P_{\mathrm{ap}}+c_{\mathrm{vp}} P_{\mathrm{vp}}$ is constant. In order to model hemorrhage we have to include a flow term $-F_{\text {hemorrhage }}$ in the equation describing the compartment where the loss of blood occurs [10]. If one wants to model the reaction of the CVS to orthostatic stress (HUT, LBNP) then one has to be able to distinguish between supine and upright position. This can be done by introducing upper and lower arterial systemic as well as venous systemic compartments $[14,64,65]$.

It should be observed that the modeling efforts described above considered non-pulsatile blood flow. Pulsatility can be obtained by considering the ventricles as compliance vessels with time varying compliance (in this context one usually considers the elastance of the ventricle which is the reciprocal of the compliance) and inflow and outflow valves (see [27-29]. For interaction of pulsatility and baroceptor dynamics see [92-94] and $[26,66]$.

\section{Challenges}

Modeling of the CVRS (and other physiological systems) pose a number of challenges for the mathematical components of the modeling process which became even more challenging because of the developments during recent past. These developments are characterized by increasing demands on accuracy of simulations which requires more comprehensive models. This leads to models with large numbers of parameters which makes validation of such models considerably more difficult. This situation usually is dramatically aggravated by a scarcity of data available for the specific area which is topic of the modeling process. An additional difficulty arises if at least some of the parameters have to be identified rather precisely, as is discussed in Subsection 5.1. In order to meet these requirements progress in areas like experimental design, parameter estimation etc. is needed. We discuss some recent progress in these areas in Subsection 5.2 and list some areas for future research in Subsection 5.3.

\subsection{Patient specific models}

When it comes to applications of mathematical models in a clinical context it is absolutely necessary to adapt the model to individual patients. In case of models which consider the 3-dimensional extension of components of the CVRS the patient specific geometry of this component has to be included in the model 
(see also the statements at the end of the first paragraph in Subsection 3.2). Concerning the parameters of a model it is in general hopeless to identify all parameters for an individual patient. Instead we have to determine those parameters which on the basis of available data can be identified with sufficient accuracy and which allow to distinguish between individual patients, possibly for patients in a subgroup of the total patient population. The remaining parameters should be less influential on the dynamics of the considered system and have to be determined with much less accuracy (which may be difficult enough). What we need are systematic methodologies which allow to determine those parameters which with the given data can be identified with a given accuracy. The questions concerned with patient specific models are getting more and more attention (see [50]).

\subsection{Model validation and parameter estimation}

Basis for the methodologies discussed in this subsection is a so-called output model corresponding to the given mathematical model, which is of the form

$$
y(t)=f(t, \theta), \quad 0 \leq t \leq T, \theta \in \mathcal{A} \subset \mathbb{R}^{p},
$$

where $y(t) \in \mathbb{R}^{k}$ is the vector of measurable outputs of the system, $\mathcal{A}$ is the set of admissible parameters and $T$ is the length of the observation interval. For simplicity of presentation we assume that $k=1$, i.e., we have just one measurable scalar output. Associated with the output model is a statistical model which describes the available measurements. For sampling times $0 \leq t_{1}^{N}<\cdots<t_{N}^{N} \leq T$ it is assumed that we have measurements $\xi_{j}^{N}, j=1, \ldots, N$, such that

$$
\xi\left(t_{j}^{N}\right)=f\left(t_{j}^{N}, \theta_{0}\right)+\epsilon\left(t_{j}^{N}\right), \quad j=1, \ldots, N .
$$

Here $\theta_{0} \in \mathcal{A}$ is the so-called nominal or true parameter and $\epsilon(t), 0 \leq t \leq T$, is the measurement error associated with a measurement at time $t$, which is assumed to be a representation of a random variable $\mathcal{E}(t)$. The stochastic process $\mathcal{E}(\cdot)$ is called the measurement noise on $[0, T]$. Then equation (5.2) shows that also $\xi\left(t_{j}^{N}\right)$ is a representation of a random variable $\Xi\left(t_{j}^{N}\right)$. The process $\Xi(\cdot)$ is the observation process. Instead of (5.2) we can also take

$$
\Xi\left(t_{j}^{N}\right)=f\left(t_{j}^{N}, \theta_{0}\right)+\mathcal{E}\left(t_{j}^{N}\right), \quad j=1, \ldots, N,
$$

as the statistical model. The standard assumptions on the noise process are that the random variables $\mathcal{E}(t), 0 \leq t \leq T$, are i.i.d. with expected value 0 and variance $\operatorname{Var} \mathcal{E}(t)=\sigma(t)^{2}$ (compare [36, 84]). The assumption that the $\mathcal{E}\left(t_{j}^{N}\right)$ are independent may be not satisfied, for instance, in cases where the sampling times are close to each other. In such a case one has to consider correlated measurements (see [91]).

In order to simplify notation we introduce the vectors $X=\left(\xi\left(t_{1}^{N}\right), \ldots, \xi\left(t_{N}^{N}\right)\right)^{\top}, F(\theta)=$ $\left(f\left(t_{1}^{N}, \theta\right), \ldots, f\left(t_{N}^{N}, \theta\right)\right)^{\top}$. Given the statistical model (5.2) a very common approach to the parameter estimation problem is to obtain the parameter estimate $\hat{\theta}$ as the minimizer of a quadratic (in the residuals) cost functional

$$
\begin{aligned}
\hat{\theta} & =\underset{\theta \in \mathcal{A}}{\operatorname{argmin}} J(X, F(\theta)), \quad \text { where } \\
J(X, F(\theta)) & =\sum_{j=1}^{N} \frac{1}{\sigma\left(t_{j}^{N}\right)^{2}}\left(\xi\left(t_{j}^{N}\right)-f\left(t_{j}^{N}, \theta\right)\right)^{2}, \quad \theta \in \mathcal{A} .
\end{aligned}
$$

It is important to note that $\hat{\theta}$ is a representation of a random vector $\hat{\Theta}$ which is called the least squares estimator of our parameter estimation problem. For other approaches to the parameter estimation problem see $[36,84]$. A very interesting approach to parameter estimation is to use Kalman filtering which allows also time-dependent parameters (see [4]). Of course, the statistical properties of $\hat{\Theta}$ are 
important. Using asymptotic theory (asymptotic in the sense of large numbers of measurements) and linearization of the output model around $\theta_{0}$ we obtain (see [84, Chapter 12], for instance)

$$
\mathrm{E}(\hat{\Theta}) \approx \theta_{0}, \quad \operatorname{Cov}(\hat{\Theta}) \approx \mathcal{F}\left(\theta_{0}\right)^{-1}
$$

Here $\mathrm{E}(\cdot)$ denotes the expected value and $\mathcal{F}(\theta)$ is the Fisher information matrix corresponding to our parameter estimation problem, which is given as

$$
\mathcal{F}(\theta)=\left(\frac{\partial F}{\partial \theta}(\theta)\right)^{\top} D \frac{\partial F}{\partial \theta}(\theta) \in \mathbb{R}^{p \times p}
$$

where $D=\operatorname{diag}\left(1 / \sigma\left(t_{1}^{N}\right)^{2}, \ldots, 1 / \sigma\left(t_{N}^{N}\right)^{2}\right)$. The $N \times p$-matrix $S(\theta)=(\partial F / \partial \theta)(\theta)$ is called the sensitivity matrix for the output model (5.1). It should be noted that the matrices $\mathcal{F}(\theta)$ and $S(\theta)$ not only depend on $\theta \in \mathcal{A}$ but also on the sampling mesh $t_{1}^{N}, \ldots, t_{N}^{N}$. The Fisher information matrix is of central importance in parameter estimation problems. For instance, it is very important to assess the quality of a parameter estimate $\hat{\theta}$. A measure for this are the standard errors of the components of $\hat{\theta}_{i}, \operatorname{SE}\left(\hat{\theta}_{i}\right)=\left(\operatorname{Var}\left(\hat{\theta}_{i}\right)\right)^{1 / 2}$. Under certain assumptions (see [84, Chapter 12]) we have the asymptotic formula

$$
\mathrm{SE}\left(\hat{\theta}_{i}\right) \approx \sqrt{\left(\mathcal{F}\left(\theta_{0}\right)^{-1}\right)_{i, i}}, \quad i=1, \ldots, p
$$

Optimal experimental design refers to choose the data acquisition procedure (observation interval, number of measurements and location of sampling times, for instance) according to minimizing a design criterion [70]. The design criteria are continuous functions of the Fisher information matrix $\mathcal{F}\left(\theta_{0}\right)$, as for instance $1 / \operatorname{det} \mathcal{F}\left(\theta_{0}\right)$ (D-optimal design) or $\lambda_{\max } \mathcal{F}^{-1}\left(\theta_{0}\right)$ (E-optimal design) (compare [16,31,81]). In [7] the design criterion

$$
\sum_{i=1}^{p}\left(1 / \theta_{0, i}^{2}\right)\left(\mathcal{F}\left(\theta_{0}\right)^{-1}\right)_{i, i}
$$

was introduced in order to define SE-optimal designs in the context of very general sampling strategies characterized by probability measures on the sampling interval. See [8] for a comparison of D-optimal, E-optimal and SE-optimal designs and [9] for a Monte Carlo based analysis.

The problems discussed in Subsection 5.1 require to select those parameters of a model for which the parameter estimation problem is well-posed. The methodologies in this context are termed parameter subset selection methods. Presently there are two approaches available in the literature. One is based on a quadratic approximation of the cost functional $J(X, F(\theta))$ given by

$$
J(X, F(\theta))=J\left(X, F\left(\theta_{0}\right)+\left(X-F\left(\theta_{0}\right)\right)^{\top} D S\left(\theta_{0}\right) \delta \theta+\frac{1}{2} \delta \theta^{\top} \mathcal{F}\left(\theta_{0}\right) \delta \theta, \quad \delta \theta=\theta-\theta_{0},\right.
$$

in a neighborhood of $\theta_{0}$ which is obtained by neglecting the second order derivatives in the Hessian of $J$ (as is done when a Newton procedure is reduced to Gauss-Newton). The minimizer of this quadratic approximation is a solution of the following equation (first order condition):

$$
\mathcal{F}\left(\theta_{0}\right) \delta \theta=-S\left(\theta_{0}\right)^{\top} D\left(X-F\left(\theta_{0}\right)\right) .
$$

The idea is to choose those components of the parameter vector (respectively those rows and columns of $\left.\mathcal{F}\left(\theta_{0}\right)\right)$ such that the reduced system is well-posed. This is accomplished by using a rank-revealing QR-factorization (see $[33,38,79]$ ).

Another approach is using the criterion (5.7) which amounts to a combinatorial search of the best $k$ parameters among the given $p$ parameters (see [6]). 


\subsection{Future developments}

All the results presented in this section are local in the sense that the choice of the nominal parameter vector $\theta_{0}$ is of crucial importance. This is also true for most results concerning sensitivity analysis available in the literature. Future research will have to consider results on sensitivity analysis, experimental design and parameter subset selection being valid not for a specific nominal parameter vector but for a sufficiently large subdomain of $\mathcal{A}$, the domain of admissible parameters.

Another important topic for future research is to link methods of time series analysis (which have not been discussed in this survey) and physiology based modeling.

\section{References}

[1] V. Agoshkov, A. Quarteroni, G. Rozza. Shape design in aorto-coronaric bypass anastomoses using perturbation theory. SIAM Journal on Numerical Analysis, 44 (2006), 367-384.

[2] J. Alastruey, K. H. Parker, J. Peiró, S. Sherwin. Lumped parameter outflow models for 1-D blood flow simulations: Effect on pulsewaves and parameter estimation. Communications in Computational Physics, 4 (2008), 2-19.

[3] J. Alfon, T. Royo, X. Garcia-Moll, L. Badimon. Platelet deposition on eroded vessel walls at a stenotic shear rate is inhibited by lipid-lowering treatment with atorvastatin. Arterioscler. Thromb. Vasc. Biol., 19 (1999), $1812-1817$.

[4] A. Attarian, J. Batzel, B. Matzuka, H. T. Tran. Application of the unscented Kalman filtering to parameter estimation. Mathematical Model Development and Validation in Physiology: Application to the Cardiovascular and Respiratory Systems, J. J. Batzel, M. Bachar, and F. Kappel, eds., vol. 2064 of Lecture Notes in Mathematics, Berlin, 2012, Springer-Verlag. to appear.

[5] E. O. Attinger. The physics of pulsatile blood flow with particular reference to small vessels. Investigative Ophthalmology, 4 (1965), 973-987.

[6] H. T. Banks, A. Cintrón-Arias, F. Kappel. Parameter selection methods in inverse problem formulation. Mathematical Modeling and Validation in Physiology: Application to the Cardiovascular and Respiratory Systems, J. J. Batzel, M. Bachar, F. Kappel, eds., vol. 2064 of Lecture Notes in Mathematics, Berlin, 2012, Springer-Verlag. to appear.

[7] H. T. Banks, S. Dediu, S. Ernstberger, F. Kappel. Generalized sensitivities and optimal experimental design. J. Inverse and Ill-Posed Problems, 18 (2010), 25-83.

[8] H. T. Banks, K. Holm, F. Kappel. Comparison of optimal design methods in inverse problems. Inverse Problems, 27 (2011).

[9] H. T. Banks, K. Holm, F. Kappel. A Monte Carlo based analysis of optimal design criteria. J. Inverse and Ill-Posed Problems, 20 (2012), 1-38.

[10] J. Batzel, M. Fink, F. Kappel. Modeling the human cardiovascular-respiratory control response to blood volume loss due to hemorrhage. Positive Systems. C. Commault, N. Marchand, eds., vol. 341 of Lecture Notes in Control and Information Sciences, Berlin, 2006, Springer-Verlag, 145-152.

[11] J. J. Batzel, M. Bachar, V. Bhalani, F. Kappel, P. Kotanko, J. Raiman. Haemodynamics, Chapter 10, "Mathematical Physiology" (A. De Gaetano and P. Palumbo, Eds.), Encyclopedia of Life Support Systems (EOLSS), Eolss Publishers, Oxford, UK, 2008.

[12] J. J. Batzel, M. Bachar, F. Kappel. The Circulatory System. Chapter 9, "Mathematical Physiology" (A. De Gaetano and P. Palumbo, Eds.), Encyclopedia of Life Support Systems (EOLSS), Eolss Publishers, Oxford, UK, 2008.

[13] J. J. Batzel, M. Bachar, F. Kappel. Respiration and Gas Exchange, Chapter 12, "Mathematical Physiology" (A. De Gaetano and P. Palumbo, Eds), Encyclopedia of Life Support Systems (EOLSS), Eolss Publishers, Oxford, UK, 2008.

[14] J. J. Batzel, N. Goswami, H. K. Lackner, A. Roessler, M. Bachar, F. Kappel, H. Hinghofer-Szalkay. Patterns of cardiovascular control during repeated tests of orthostatic loading. Cardiovascular Engineering: An international Journal, 9 (2009), 134-143.

[15] J. J. Batzel, F. Kappel, D. Schneditz, H. T. Tran. Cardiovascular and Respiratory Systems: Modeling, Analysis and Control. vol. 34 of Frontiers in Applied Mathematics, SIAM, Philadelphia, 2007.

[16] M. P. F. Berger, W. K. Wong, eds., Applied Optimal Designs, John Wiley \& Sons, Chichester, UK, 2005.

[17] M. J. Bishop, G. Plank, E. Vigmond. Investigating the role of the coronary vasculature in the mechanisms of defibrillation. Circ Arrhythm Electrophysiol, 5 (2012), 210-219.

[18] A. Brunberg, S. Heinke, J. Spillner, R. Autschbach, D. Abel, S. Leonhardt. Modeling and simulation of the cardiovascular system: a review of applications, methods, and potentials. Biomed. Tech., 54 (2009), 233-244.

[19] S. Cavalcanti, S. Cavani, A. Ciandrini, G. Avanzolini. Mathematical modeling of arterial pressure response to hemodialysis-induced hypovolemia. Computers in Biology and Medicine, 36 (2006), 128-144.

[20] S. Cavalcanti, S. Cavani, A. Santoro. Role of short-term regulatory mechanisms on pressure response to hemodialysisinduced hypovolemia. Kidney International, 61 (2002), 228-238.

[21] S. Cavalcanti, A. Ciandrini, S. Severi, F. Badiali, S. Bini, A. Gattiani, L. Cagnoli, A. Santoro. Model-based study of the effects of the hemodialysis technique on the compensatory response to hypovolemia, Kidney International, 65 (2004), 1499-1510.

[22] S. Cavalcanti, L. Y. Di Marco. Numerical simulation of the hemodynamic response to hemodialysis-induced hypovolemia. Artif. Organs, 23 (1999), 1063-1073. 
[23] S. Cavani, S. Cavalcanti, G. Avanzolini. Model based sensitivity analysis of arterial pressure response to hemodialysisinduced hypovolemia. ASAIO Journal, 2001 (2001), 377-388.

[24] P. Crosetto, S. Deparis, G. Fourestey, A. Quarteroni. Parallel algorithms for fluid-structure interaction problems in haemodynamics. SIAM Journal on Scientific Computing, 33 (2011), 1598-1622.

[25] C. D'Angelo, A. Quarteroni. On the coupling of 1d and 3d diffusion-reaction equations: Application to tissue perfusion problems. Mathematical Models and Methods in Applied Sciences, 18 (2008), 1481-1504.

[26] M. Danielsen, J. T. Ottesen. A dynamical approach to the baroreceptor regulation of the cardiovascular system. Proceeding to the 5th International Symposium, Symbiosis '97, 1997, 25 - 29.

[27] M. Danielsen, J. T. Ottesen. Describing the pumping heart as a pressure source. J. Theor. Biol., 212 (2001), 71-81.

[28] A. de los Reyes V, F. Kappel. Modeling pulsatility in the human cardiovascular system. Mathematica Balcanica, New Series, 24 (2010), 229-242.

[29] A. A. de los Reyes V. A mathematical model for the cardiovascular system with a measurable pulsatile pressure output. PhD thesis, University of Graz, Graz (Austria), March 2010.

[30] R. Fåhræus, T. Lindqvist. The viscosity of blood in narrow capillary tubes. Am. J. Physiol., 96 (1931), $562-568$.

[31] V. V. Fedorov, P. Hackel. Model-Oriented Design of Experiments. Springer-Verlag, New York, NY, 1997.

[32] G. D. Fink. Hypothesis: the systemic circulation as a regulated free-market economy. A new approach for understanding the long-term control of blood pressure. Clin. Exp. Pharmacol. Physiol., 32 (2005), 377-383.

[33] M. Fink, A. Attarian, H. T. Tran. Subset selection for parameter estimation in an hiv model. Proc. Applied Math. and Mechanics, 7 (2008), 11212,501-11221,502.

[34] A. Fishman, N. Cherniack, J. Widdicombe, A. P. Society, Handbook of Physiology: A Critical, Comprehensive Presentation of Physiological Knowledge and Concepts. The respiratory system. Control of breathing, / volume editors, Neil S. Cherniack, John G. Widdicombe / executive editor, Stephen R. Geiger, American Physiological Society, 1986.

[35] L. Formaggia, J. F. Gerbeau, F. Nobile, A. Quarteroni. On the coupling of 3D and 1D Navier-Stokes equations for flow problems in compliant vessels. Computer Methods in Applied Mechanics and Engineering, 191 (2001), 561-582.

[36] G. C. Goodwin, R. L. Payne. Dynamic System Identification, Experimental Design and Data Analysis. vol. 136 of Mathematics in Science and Engineering, Academic Press, New York, 1977.

[37] N. Goswami, H. Lackner, I. Papousek, J. P. Montani, D. D. Jezova, H. Hinghofer-Szalkay. Does mental arithmetic before head up tilt have an effect on the orthostatic cardiovascular and hormonal responses. Acta Astronautica, 68 (2011), 1589-1594.

[38] D. M. Gu, S. C. Eisenstat. Efficient algorithms for computing a strong rank-revealing QR factorization. SIAM J. Sci. Comput., 17 (1996), 848-869.

[39] A. C. Guyton, Textbook of Medical Physiology, W. B. Saunders Company, Philadelphia, Pa, 8 ed., 1991.

[40] A. C. Guyton, J. E. Hall, Guyton Hall Textbook of Medical Physiology, Saunders/Elsevier, Philadelphia, Pa, 11 ed., 2005.

[41] M. Habib. Control of the Human Cardiovascular-Respiratory System under a Time-Varying Ergonometric Workload. $\mathrm{PhD}$ thesis, University of Graz, Graz (Austria), May 2011.

[42] T. Heldt, E. B. Shim, R. D. Kamm, R. G. Mark. Computational modeling of cardiovascular response to orthostatic stress. J. Appl. Physiol., 92 (2002), 1239-1254.

[43] F. C. Hoppensteadt, C. S. Peskin. Mathematics in Medicine and the Life Sciences. vol. 10 of Texts in Applied Mathematics, Springer Verlag, New York, NY, 1992.

[44] F. Kappel, J. J. Batzel. Survey of research in modeling the human respiratory and cardiovascular systems. Research Directions in Distributed Parameter Systems, R. C. Smith and M. A. Demetriou, eds., vol. 27 of Frontiers in Applied Mathematics, SIAM, Philadelphia, Pa, 2003, ch. 8, 187-218.

[45] F. Kappel, M. Fink, J. Batzel. Aspects of control of the cardiovascular-respiratory system during orthostatic stress induced by lower body negative pressure. Math. Biosciences, 206 (2007), 273-308.

[46] F. Kappel, S. Lafer, R. O. Peer. A model for the cardiovascular system under an ergometric workload. Surv. Math. Ind., 7 (1997), 239-250.

[47] F. Kappel, R. O. Peer. A mathematical model for fundamental regulation processes in the cardiovascular system. J. Math. Biol., 31 (1993), 611-631.

[48] J. Keener, J. Sneyd, Mathematical Physiology, Vol II: Systems Physiology, vol. 8 of Interdisciplinary Applied Mathematics, Springer Verlag, New York, 2nd ed., 2008.

[49] T. Kenner. Physiology of circulation. Cardiology, 1st ed., S. D. Volta, E. Braunwald, A. B. D. Luna, V. Jezek, M. L. Brochier, S. A. Mortensen, F. Dienstl, P. A. Poole-Wilson, eds., Clinical Medicine, New York, 1999, McGraw-Hill, $15-25$.

[50] R. C. P. Kerckhoffs, ed.. Patient-Specific Modeling of the Cardiovascular System, Technology-Driven Personalized Medicine, Springer-Verlag, New York, 2010.

[51] A. S. Kholodov, S. S. Simakov, A. V. Evdokimov, Y. A. Kholodov. Matter transport simulations using $2 D$ model of peripheral circulation coupled with the model of large vessels. Proc. II Int. Conf. On Comput. Bioeng., September 14-16, Lisbon, H. Rodrigues, M. Cerrolaza, M. Doblaré, J. Ambrósio, and M. Viceconti, eds., vol. 1, Lisbon, 2005 , IST Press, 479-490.

[52] R. E. Klabunde. Cardiovascular Physiology Concepts. Lippincott Williams \& Wilkins, Baltimore, Md, 2005.

[53] P. Kuijper, H. G. Torres, J.-W. Lammers, J. Sixma, L. Koenderman, J. Zwaginga. Platelet and fibrin deposition at the damaged vessel wall: Cooperative substrates for neutrophil adhesion under flow conditions. Blood, 89 (1997), 166-175.

[54] J. R. Levick. An Introduction to Cardiovascular Physiology. Oxford Univ. Press, New York, 4th ed., 2003. 
[55] S. L. Mabry, L. F. Bic, K. M. Baldwin. CVSys: a coordination framework for dynamic and fully distributed cardiovascular modeling and simulation. Biomedical Sensing and Imaging Technologies, R. A. Lieberman and T. Vo-Dinh, eds., vol. 3253 of Proc. SPIE, 1998, 208-218.

[56] R. Mittal, G. Iaccarino. Immersed boundary methods. Annual Rev. Fluid Mech., 37 (2005), 239-261.

[57] M. E. C. Mutsaers, M.Bachar, J. J. Batzel, F. Kappel, S. Volkwein. Receding horizon controller for the baroreceptor loop in a model for the cardiovascular system. Cardiovascular Engineering: An international Journal, 8 (2008), 14-22.

[58] S. Muzdeka, E. Barbieri. Control theory inspired considerations of the mathematical models of defibrillation. Proceedings of the 44th IEEE Conference on Decision and Control, and the European Control Conference 2005, IEEE Conderence Publications, 2005, 7416-7421.

[59] S. Neumann. Modeling Acute Hemorrhage in the Human Cardiovascular System. PhD thesis, University of Pennsylvania, Pensylvania, 1996.

[60] P. Novak, V. Novak, J. Spies, V. Gordon, T. Lagerlund, G. Petty. Evaluation of cerebral autoregulation in orthostatic hypotension and POTS. Clin. Auton. Res., 7 (1997), p. 238.

[61] V. Novak, P. Novak, J. M. Spies, P. A. Low. Autoregulation of cerebral blood flow in orthostatic hypotension. Stroke, 29 (1998), 104-111.

[62] M. S. Olufsen. Modeling flow and pressure in systemic arteries. Applied Mathematical Models in Human Physiology, J. T. Ottesen, M. Olufsen, and J. K. Larsen, eds., SIAM Monographs on Mathematical Modeling and Computation, SIAM, Philadelphia, Pa, 2004, ch. 5, 91-136.

[63] M. S. Olufsen, A. Nadim, L. A. Lipsitz. Dynamics of cerebral blood flow regulation explained using a lumped parameter model. Am. J. Physiol., 282 (2002), R611-R622.

[64] M. S. Olufsen, J. T. Ottesen, H. T. Tran. Modeling cerebral blood flow control during posture change from sitting to standing. J. Cardiov. Eng., 4 (2004), 47-58.

[65] M. S. Olufsen, J. T. Ottesen, H. T. Tran, L. M. Ellwein, L. A. Lipsitz, V. Novak. Blood pressure and blood flow variation during postural change from sitting to standing: Model development and validation. J. Appl. Physiol., 99 (2005), 1523-1537.

[66] J. T. Ottesen. Modelling the baroreflex-feedback mechanism with time-delay. J. Math. Biol., 36 (1997), 41-63.

[67] J. T. Ottesen, M. Danielsen, eds., Mathematical Modelling in Medicine, vol. 71 of Studies in Health Technology and Informatics, IOS Press, Amsterdam, 2000.

[68] J. T. Ottesen, M. S. Olufsen, J. K. Larsen, eds., Applied Mathematical Models in Human Physiology, Monographs on Mathematical Modeling and Computation, SIAM, Philadelphia, 2004.

[69] T. Passerini, M. de Luca, L. Formaggia, A. Quarteroni, A. Veneziani. A 3D/1D geometrical multiscale model of cerebral vasculature. Journal of Engineering Mathematics, 64 (2009), 319 - 330.

[70] A. Pázman. Foundations of Optimum Experimental Design, Mathematics and Its Applications. D. Reidel Publ. Comp., Dordrecht, 1986.

[71] K. Perktold, M. Hofer, G. Rappitsch, M. Loew, B. D. Kuban, M. H. Friedman. Validated computation of physiologic flow in a realistic coronary artery branch. J. Biomech., 31 (1998), 217-228.

[72] K. Perktold, G. Rappitsch. Mathematical modeling of arterial blood flow and correlation to atherosclerosis. Technol. Health Care, 3 (1995), 139 - 151.

[73] C. S. Peskin. Flow Patterns around Heart Valves. PhD thesis, Albert Einstein College of Medicine, New York, 1972.

[74] C. S. Peskin, D. M. McQueen. Modeling prosthetic heart valves for numerical analysis of blood flow in the heart. J. Comput. Phys., 37 (1980), 113-132.

[75] C. S. Peskin, D. M. McQueen. Cardiac fluid dynamics. High-performance Computing in Biomedical Research, T. C. Pilkington et al., ed., CRC Press, Boca Raton, 1993.

[76] C. S. Peskin, D. M. McQueen. Mechanical equilibrium determines the fractal fiber architecture of aortic heart valve leaflets. Am. J. Physiol., 266 (1994), H319-H328.

[77] C. S. Peskin, D. M. McQueen. Fluid dynamics of the heart and its valves, in Case Studies in Mathematical Modeling - Ecology, Physiology, and Cell Biology, H. G. Othmer, F. R. Adler, M. A. Lewis, J. C. Dallon, eds., Prentice Hall, Englewood Cliffs, New Jersey, 1996, ch. 14, 309-337.

[78] C. S. Peskin, B. F. Printz. Improved volume conservation in the computation of flows with immersed boundaries. J. Comput. Phys., 105 (1993), 33-46.

[79] S. R. Pope, L. M. Ellwein, C. L. Zapata, V. Novak, C. T. Kelley, M. S. Olufsen. Estimation and identification of parameters in a lumped cerebrovascular model. Mathematical Biosciences and Engineering, 6 (2009), 93-115.

[80] M. Prosi, P. Zunino, K. Perktold, A. Quarteroni. Mathematical and numerical models for transfer of low-density lipoproteins through the arterial walls: A new methodology for the model set up with applications to the study of disturbed lumenal flow. Journal of Biomechanics, 38 (2005), 903-917.

[81] F. Pukelsheim. Optimal Design of Experiments. JohnWiley \& Sons, New York, NY, 1993.

[82] A. Quarteroni, A. Veneziani, P. Zunino. Mathematical and numerical modeling of solute dynamics in blood flow and arterial walls. SIAM Journal on Numerical Analysis, 39 (2001), 1488 - 511.

[83] L. B. Rowell. Human Cardiovascular Control. Oxford University Press, New York, 1993.

[84] G. A. F. Seber, C. J. Wild, Nonlinear Regression. Wiley Series in Probability and Mathematical Statistics. J. Wiley, New York, 1989.

[85] B. W. Smith, J. G. Chase, G. M. Shaw, R. I. Nokes. Experimentally verified minimal cardiovascular system model for rapid diagnostic assistance, Control Engineering Practice, 13 (2005), 1183-1193.

[86] J. Smith, J. Kampine. Circulatory Physiology. Williams, Wilkins, Baltimore, 1990. 
[87] W.-B. Tay, Y.-H. Tseng, L.-Y. Lin, W.-Y. Tseng. Towards patient-specific cardiovascular modeling system using the immersed boundary technique. BioMedical Engineering OnLine, 10 (2011).

[88] W. D. Timmons. Cardiovascular models and control. "The Biomedical Engineering Handbook" (Chapter 160), J. D. Branzino, ed., Boca Raton, 2000, CRC Press LLC.

[89] N. Trayanova, G. Plank, B. Rodríguez. What have we learned from mathematical models of defibrillation and postshock arrhythmogenesis? Application of bidomain simulations. Heart Rhythm, 3 (2006), 1232-1235.

[90] R. F. Tuma, W. N. Duràn, K. Ley, eds.. Microcirculation. Elsevier, Amsterdam, 2 ed., 2008.

[91] D. Ucinski, A. Atkinson. Experimental design for time-dependent models with correlated observations. Studies in Nonlinear Dynamics and Econometrics, 8 (2004).

[92] M. Ursino. Interaction between carotid baroregulation and the pulsating heart: A mathematical model. Am. J. Physiol., 275 (1998), H1733-H1747.

[93] M. Ursino. A mathematical model of the carotid baroregulation in pulsating conditions. IEEE Trans. Biomed. Eng., 46 (1999), 382-392.

[94] M. Ursino, A. Fiorenzi, E. Belardinelli. The role of pressure pulsatility in the carotid baroreflex control: A computer simulation study. Comput. Biol. Med., 26 (1996), 297-314.

[95] M. Ursino, M. Innocenti. Mathematical investigation of some physiological factors involved in hemodialysis hypotension. Artif. Organs, 21 (1997), 891-902.

[96] M. Ursino, M. Innocenti. Modeling arterial hypotension during hemodialysis. Artif. Organs, 21 (1997), 873-890.

[97] F. Vadakkumpadan, L. J. Rantner, B. Tice, P. Boyle, A. J. Prassl, E. Vigmond, G. Plank, N. Trayanova. Image-based models of cardiac structure with applications in arrhythmia and defibrillation studies. J Electrocardiol, 42 (2009), 157.e1-157.10.

[98] N. Westerhof, N. Stergiopulos. Models of the arterial tree. Mathematical Modelling in Medicine, J. T. Ottesen, M. Danielsen, eds., vol. 71 of Studies in Health Technology and Informatics, Amsterdam, The Netherlands, 2000, IOS Press, 65-78.

[99] N. Westerhof, N. Stergiopulos, M. I. M. Noble. Snapshots of Hemodynamics. vol. 18 of Basic Science for the Cardiologist, Kluwer Academic Publishers, Dordrecht, 2005. 